\title{
Variability of Human Corticospinal Excitability Tracks the State of Action Preparation
}

\author{
Miriam C. Klein-Flügge, ${ }^{1}$ David Nobbs, ${ }^{1}$ Julia B. Pitcher, ${ }^{2}$ and Sven Bestmann ${ }^{1}$ \\ ${ }^{1}$ Sobell Department of Motor Neuroscience and Movement Disorders, UCL Institute of Neurology, University College London, London WC1 N3BG, United \\ Kingdom, and ${ }^{2}$ Robinson Institute, School of Paediatrics and Reproductive Health, University of Adelaide, Adelaide SA 5005, Australia
}

Task-evoked trial-by-trial variability is a ubiquitous property of neural responses, yet its functional role remains largely unclear. Recent work in nonhuman primates shows that the temporal structure of neural variability in several brain regions is task-related. For example, trial-by-trial variability in premotor cortex tracks motor preparation with increasingly consistent firing rates and thus a decline in variability before movement onset. However, whether noninvasive measures of the variability of population activity available from humans can similarly track the preparation of actions remains unknown. We tested this by using single-pulse transcranial magnetic stimulation (TMS) over primary motor cortex (M1) to measure corticospinal excitability (CSE) at different times during action preparation. First, we established the basic properties of intrinsic CSE variability at rest. Then, during the task, responses (left or right button presses) were either directly instructed (forced choice) or resulted from a value decision (choice). Before movement onset, we observed a temporally specific task-related decline in CSE variability contralateral to the responding hand. This decline was stronger in fastresponse compared with slow-response trials, consistent with data in nonhuman primates. For the nonresponding hand, CSE variability also decreased, but only in choice trials, and earlier compared with the responding hand, possibly reflecting choice-specific suppression of unselected actions. These findings suggest that human CSE variability measured by TMS over M1 tracks the state of motor preparation, and may reflect the optimization of preparatory population activity. This provides novel avenues in humans to assess the dynamics of action preparation but also more complex processes, such as choice-to-action transformations.

\section{Introduction}

Cortical neural activity elicited by the same stimulus or process exhibits large trial-by-trial variability (Shadlen and Newsome, 1998), which is thought to arise from the stochastic nature of the neuronal firing process (Rosner and Warzecha, 2011). Recent work in animals has found that neural variability is, in turn, a likely source of variability in motor responses (Churchland et al., 2006b; Faisal et al., 2008; Miller and Katz, 2010), but can also track the functional state of the motor system. For example, the variability of neuronal firing rates recorded from primate premotor cortex decreases before executing a prepared response, despite little change in mean firing rate (Churchland et al., 2006a). Furthermore, when firing rates are more variable, monkeys take longer to execute the required action (Churchland et al., 2006a).

One theory is that this increase in firing rate consistency (and thus lower variability) during the evolving action plan reflects an optimization process that brings firing rates from their initial

\footnotetext{
Received May 17, 2012; revised Jan. 22, 2013; accepted Feb. 8, 2013

Author contributions: M.C.K.-F. and S.B. designed research; M.C.K.-F., D.N., and J.B.P. performed research; M.C.K.-F. analyzed data; M.C.K.-F. and S.B. wrote the paper.

This work was supported by the Wellcome Trust (086120/Z08/Z), the European Research Council (ActSelectContext, 260424), the Biotechnology and Biological Sciences Research Council, and the Australian National Health and Medical Research Council (ID 299087). We thank Ashleigh Smith and Joanna Cole for their help in collecting the data at rest.

The authors declare no competing financial interests.

Correspondence should be addressed to Miriam C. Klein-Flügge, Sobell Department of Motor Neuroscience and Movement Disorders, 33 Queen Square, WC1N 3BG London, UK. E-mail: m.klein@ucl.ac.uk.

DOI:10.1523/JNEUROSCI.2448-12.2013

Copyright $\odot 2013$ the authors $\quad 0270-6474 / 13 / 335564-09 \$ 15.00 / 0$
}

state into a so-called "optimal subspace," defined as the subset of firing rates required to generate a sufficiently accurate movement response (Churchland et al., 2006a; Afshar et al., 2011).

Whether the variability of a population response can predict the state of action preparation, as has been reported at the singleunit level, remains unknown. Based on theoretical grounds (Sussillo and Abbott, 2009; Abbott et al., 2011) and direct neural recordings in nonhuman primates (Churchland et al., 2010a), the variability decline in neural responses during action preparation is thought to reflect a network property. If this is indeed the case, population measures of variability should similarly track the state of action preparation. Here we sought to test this theory noninvasively in humans by measuring changes in the variability of corticospinal excitability (CSE) before a movement.

We applied transcranial magnetic stimulation (TMS) to the hand representation of human primary motor cortex (M1). This elicits a motor-evoked potential (MEP) in contralateral hand muscles, which provides an index for the state of CSE of the pool of neurons thus stimulated, potentially including cortical as well as subcortical and spinal contributions. Importantly, the MEP size reflects the current responsiveness of these pathways rather than neuronal activity, which is measured in direct recordings. We first characterized the precise relationship between MEP size and variability at rest. This enabled identification of changes in variability during action preparation over and above the intrinsic changes in the CSE variability due to variations in MEP amplitude. We expected that the variability of TMS-evoked responses would show time-dependent fluctuations that reflect action 


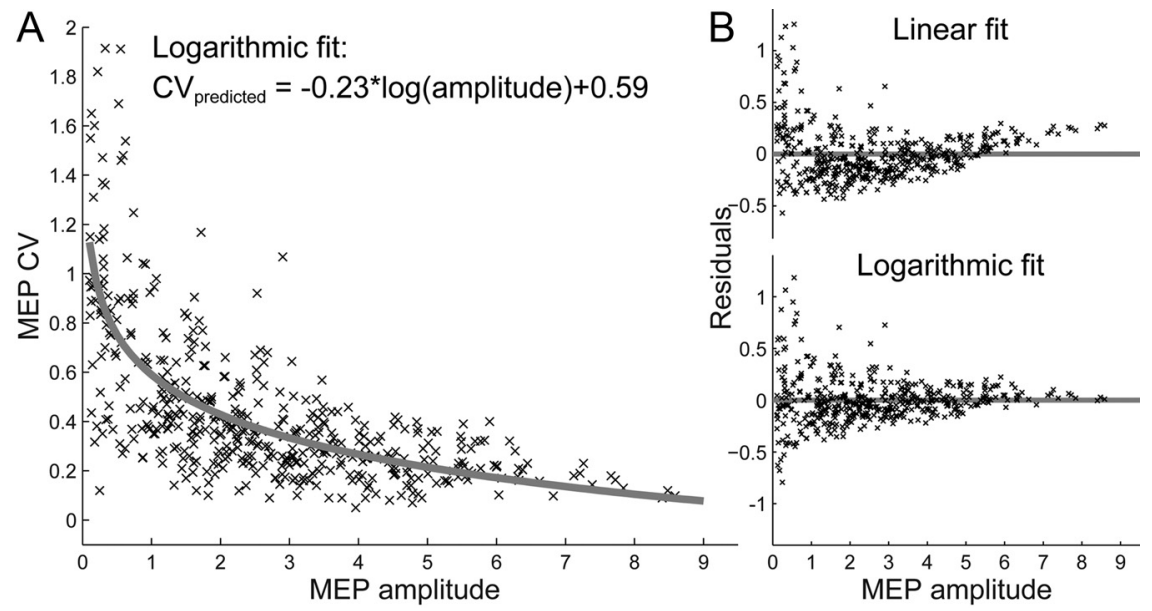

Figure 1. Relationship between MEP amplitude and variability at rest. $\boldsymbol{A}, \mathrm{MEP}$ amplitudes are plotted against the CV for all participants. The relationship between MEP amplitude and CV is well characterized by a logarithmic fit. The logarithmic function shown was obtained by fitting the merged data of all participants. $\boldsymbol{B}$, The residuals from both the linear and the logarithmic fit illustrate the superiority of the logarithmic fit.

preparation, with decreased variability closer to movement. Previous work has shown that CSE measures closely track action preparation (Mars et al., 2007; van Elswijk et al., 2007; Bestmann et al., 2008). However, whether the evolution of variability in CSE can similarly track the state of action preparation remains unknown.

In a final step, we tested whether changes in CSE variability were behaviorally relevant, and whether there was any difference between instructed and value-guided action selection. Firing-rate variability of single neurons in premotor cortex and the frontal eye fields correlates with reaction times (Churchland et al., 2006a; Cohen et al., 2007; Steinmetz and Moore, 2010; Purcell et al., 2012), thus indicating behavioral relevance of such variability changes. We tested whether this also applies to human CSE measures and, furthermore, compared CSE variability before actions in forced-choice and choice trials. To the best of our knowledge, we provide the first demonstration in humans that variability in CSE tracks action preparation.

\section{Materials and Methods}

The present study comprises two independent datasets, one acquired at rest and one acquired during a behavioral choice task. Here we report the methodological details critical for the present study. Other aspects of these data have been reported by Smith et al. (2011) and by Klein-Flügge and Bestmann (2012). All participants were right-handed, completed a TMS safety screen before enrolment in the study (Rossi et al., 2009), and gave written informed consent. Experiments were conducted with local ethics approvals and in accordance with the Declaration of Helsinki.

Experiment 1: identifying the relationship between MEP size and variability at rest

Participants and experimental procedure. Data were acquired from 46 healthy individuals (age range, 17-31 years; mean age, 21 years; 29 male; 17 female) with no history of neurological or psychiatric disorder, and with normal or corrected-to-normal vision. In all participants, TMS was applied to the left hemisphere. In 13 participants, data from the right hemisphere was also acquired on a different day.

TMS was delivered to M1 through a $90 \mathrm{~mm}$ figure-of-eight shaped coil connected to a monophasic Magstim $200^{2}$ stimulator (Magstim). The coil was positioned over the optimal position for eliciting an MEP in the first dorsal interosseous (FDI) of the hand contralateral to the stimulation. It was held tangentially to the skull with the handle oriented posteriorly at $\sim 45^{\circ}$ from the midsagittal axis, thus inducing a posterolateral to anteromedial current flow in the brain. Resting motor thresholds
(RMTs) were determined in each individual as the lowest stimulator output at which five MEPs with a minimum peak-to-peak amplitude of $50 \mu \mathrm{V}$ were evoked from the resting FDI in 10 consecutive trials. The group mean RMT was $42 \pm 1.2 \%$ of maximal stimulator output.

Surface electromyographic (EMG) recordings were obtained using bipolar $\mathrm{Ag}-\mathrm{AgCl}$ surface electrodes in a tendon-belly montage. The EMG signal was sampled at $5 \mathrm{kHz}$ (1401, Cambridge Electronic Design), bandpass filtered between 20 and $1000 \mathrm{~Hz}$ (D360, Digitimer), recorded with an additional $50 \mathrm{~Hz}$ notch filter, and analyzed offline.

Participants rested their hands and TMS was applied at different stimulation intensities. Intensities ranged from $100 \%$ RMT to either $100 \%$ of stimulator output or to a stimulation intensity at which the MEP amplitude had reached a plateau (the maximum stimulation intensity used corresponded to $190 \%$ RMT). The stimulation intensity was varied pseudorandomly in steps of $10 \%$ of RMT, with 10 stimuli being applied at every intensity. In the 13 subjects in whom data for both hemispheres was acquired, the stimulation intensity was increased incrementally in steps of 5\% of RMT with five trials per step.

Data preprocessing and statistical analyses. Trials in which background EMG activity exceeded the mean prestimulation EMG activity by $>2$ SDs were discarded offline. All bins with $>5$ measurements were included if the average MEP exceeded $0.1 \mathrm{mV}$. For each bin, the mean and coefficient of variation $(\mathrm{CV}=\mathrm{SD} /$ mean $)$ of the MEPs were calculated. Bins that resulted in mean MEP amplitudes exceeding the mean of all bins by $>3$ SDs were discarded. This only affected five bins with exceptionally high average MEP size (all $>9 \mathrm{mV}$ ). The remaining raw data are shown in Figure $1 A$.

The CV was used to assess MEP variability. This approach differs from studies of task-related neural variability at the single-cell level that typically use the Fano factor, which describes the ratio of the variance to the mean spike count (e.g., Mitchell et al., 2007; Churchland et al., 2010a). In single-cell recordings from nonhuman primates, the Poisson-like distribution of spiking noise implies higher variability with higher firing rates; use of the Fano factor allows one to control for the natural scaling of variance with the mean. With TMS-evoked MEPs, by contrast, there is an upper bound to the MEP amplitude that can be elicited from stimulating M1 (i.e., when the maximum number of neurons is recruited by the TMS pulse). Therefore, we expected MEP variability to decrease for larger stimulation intensities (i.e., larger MEPs). Indeed, previous work has suggested the CV of MEPs to be inversely related to the MEP amplitude (Kiers et al., 1993; Devanne et al., 1997; Capaday et al., 1999). However, previous work has not formally characterized the MEP-CV relationship.

Here, the relationship between MEP amplitude (AMP) and CV was directly determined. We compared a linear and a logarithmic fit to the data [i.e., $\mathrm{CV}_{\text {predicted }}=a^{\star} \mathrm{AMP}+b$ and $\mathrm{CV}_{\text {predicted }}=a^{\star} \log (\mathrm{AMP})+b$ ]. This required estimation of two free parameters: the slope, $a$, and intercept, $b$. The fits were obtained using Matlab's (Mathworks) polyfit function. To test whether the data are best characterized by a linear or logarithmic function, we conducted a residual analysis (Fig. 1B) as well as a formal Bayesian model comparison using the Bayes factor (BF; Kass and Raftery, 1995).

In addition to the data obtained with the MEP amplitude cutoff criteria used here $(0.1-9 \mathrm{mV})$, we also fitted data obtained with different cutoff criteria. This was done to rule out that only the specific cutoff used and the resulting shape of the logarithmic function explained the results of Experiment 2. For the lower bound, we used 0.05, 0.1, 0.5, and $1 \mathrm{mV}$, for the upper bound, we used 5, 7, and $9 \mathrm{mV}$, and we tested all resulting combinations. The superiority of the logarithmic fit over the linear fit was robust to these changes in the range of included MEP amplitudes, and the 
resulting logarithmic fits produced the same results in Experiment 2 (see Results).

\section{Experiment 2: changes in CSE variability} during action preparation

Participants. Sixteen healthy volunteers (age range, $19-31$ years; mean age, 22 years; 11 female; 5 male) with no history of neurological or psychiatric disorder, and with normal or corrected-to-normal vision, participated in this experiment. One participant was excluded from the analyses because an insufficient number of trials remained after preprocessing.

Behavioral task. Participants performed a decision task with two types of trials, choice and forced-choice trials. In forced-choice trials (33\%), one option was presented either to the left or right (with equal probability) of a central fixation point and it was associated with a reward magnitude and a probability of obtaining this monetary reward (Fig. 2). For the purpose of the present study, magnitude and probability can be neglected and this can be regarded simply as a cue instructing the required action.

In choice trials (67\%), participants had to make a choice between two options that differed in reward magnitude and probability. These were presented to the left and right of fixation. Reward magnitudes were displayed as the length of a horizontal bar; reward probabilities were shown as numbers (Fig. 2). Participants indicated their choice via a button press with the left or right index finger, corresponding to the location of the chosen option on the screen. In forced-choice trials, participants could immediately prepare and execute the required action, while in choice trials they first had to make a comparison between the expected value of both options. Critically, however, the processes of action preparation and execution can be studied in both types of trials. The maximum allowed reaction time (RT) was $3 \mathrm{~s}$. The chosen option was briefly highlighted $(500 \mathrm{~ms})$ and the outcome presented $(500 \mathrm{~ms}$; green border, win; red border, no reward; Fig. 2).

Participants performed 216 trials of training and a total of 576 trials in the main experiment (384 choice and 192 forced-choice trials; split in 4 blocks). They were paid a maximum of $£ 2$ per block, proportional to their winnings, plus a fixed amount of $£ 12$ for participating in the study.

TMS procedure. TMS pulses were delivered through a $50 \mathrm{~mm}$ figureof-eight shaped coil connected to a monophasic Magstim $200^{2}$ stimulator (Magstim) and applied over left M1. The coil was held tangentially to the skull with the handle oriented posteriorly at $\sim 45^{\circ}$ from the midsagittal axis, thus inducing a posterolateral to anteromedial current flow in the brain. The stimulation intensity was adjusted to elicit an MEP of $\sim 1.5$ $\mathrm{mV}$ in the right FDI muscle at rest (mean stimulation intensity, $53 \pm 2 \%$ of the maximum stimulator output).

Surface EMG was recorded from the right FDI using Ag-AgCl surface electrodes in a tendon-belly montage. The EMG signal was sampled at 1 $\mathrm{kHz}$, bandpass filtered between 3 and $1000 \mathrm{~Hz}$, with an additional $50 \mathrm{~Hz}$ notch filter, fed into a CED 1902 signal conditioner (Cambridge Electronic Design), digitized using a micro $1401 \mathrm{Mk}$.II analog-to-digital converter (Cambridge Electronic Design), and stored on a PC running Spike2 (Cambridge Electronic Design).

To provide a near-continuous sampling of CSE throughout a trial, TMS was applied at six different time points between trial onset and response. However, in each trial, only a single TMS pulse was applied, giving a total of 96 trials per TMS time point. The TMS delivery times were adjusted for each participant based on their choice and forcedchoice training reaction times. This enabled us to obtain measures of CSE between trial onset and response, and thus to investigate changes in CSE during action preparation (Mars et al., 2007; van Elswijk et al., 2007; Bestmann et al., 2008; Duque and Ivry, 2009; Duque et al., 2010). In forced-choice trials, the TMS times (t1-t6) corresponded to $10 \%, 35 \%$, $50 \%, 60 \%, 70 \%$, and $80 \%$ of the participant's individual mean forced- choice RT (FC-RT), thus spanning from trial onset to response. In choice trials, the first time point, $\mathrm{t} 1$, was identical ( $10 \%$ of mean FC-RT), t 2 was set to $45 \%$ of the FC-RT, and the remaining four TMS time points were spaced equidistantly between $45 \%$ of the FC-RT and $45 \% \mathrm{FC}-\mathrm{RT}+\Delta \mathrm{RT}$, where $\Delta \mathrm{RT}$ is the RT difference between choice and forced-choice trials (Klein-Flügge and Bestmann, 2012).

Data preprocessing and statistical analyses. Data preprocessing comprised three main stages. First we conservatively discarded trials that may have spuriously biased our CV estimates. Second, we response-locked and normalized the data, thus enabling group analyses. Finally, to obtain continuous measures of MEP size and $\mathrm{CV}_{\text {observed }}$ across the trial, we applied a sliding window to the data.

EMG data were analyzed in Matlab (Mathworks). We extracted peakto-peak amplitudes of the TMS-evoked MEPs for every trial. Outlier MEPs (Grubb's test $p<0.005$ ) and those contaminated by precontraction in the target FDI muscle (absolute signal $>0.1 \mathrm{mV}$ in the $100 \mathrm{~ms}$ preceding the pulse) were discarded. Trials with MEP amplitudes $<0.1$ $\mathrm{mV}$ were also discarded, thus matching the criterion applied to the resting data.

Trials with premature $(<100 \mathrm{~ms})$ or late $(>3000 \mathrm{~ms})$ responses were discarded from the analyses. In one participant, this left a total of only $49 \%$ of trials and the participant was excluded from any further analyses. In all remaining participants, an average of $15 \pm 2 \%$ of trials were discarded (small amplitude, $5 \%$; outliers, $0.02 \%$; precontraction, $10 \%$; premature/late response, $0.1 \%$ ), leaving $494 \pm 11$ of a possible total of 576 trials for further analyses. The relatively high proportion of excluded trials with precontraction is due to the inherent variability in participant's RTs, which meant that TMS would sometimes occur immediately before, or during, the overt response and therefore coincide with its corresponding burst in EMG activity.

Because we were interested in changes in MEP size and variability leading up to the response, trials were sorted according to the time between the TMS pulse and the actual response (response-locked). This led to an almost continuous distribution of TMS times, relative to the RT in a given trial, due to the inherent variability of RTs across trials. All trials in which TMS was delivered $<75 \mathrm{~ms}$ before the response (i.e., trials with unexpectedly short RTs) were discarded, including trials where TMS was applied during or after the response. This was necessary not only to avoid ramping effects (Evarts, 1966; Starr et al., 1988; Leocani et al., 2000), but also because there were too few data for robust averaging in this time range. Similarly, in trials with unusually long RTs, TMS occurred very early with respect to the RT of that trial, and these were discarded when TMS occurred earlier than the participant's mean RT before response. Thus, the distance of all TMS times with respect to the response now ranged between [ - average $\mathrm{RT}]$ and $[-75 \mathrm{~ms}]$. To allow for averaging 


\section{A MEP amplitude}

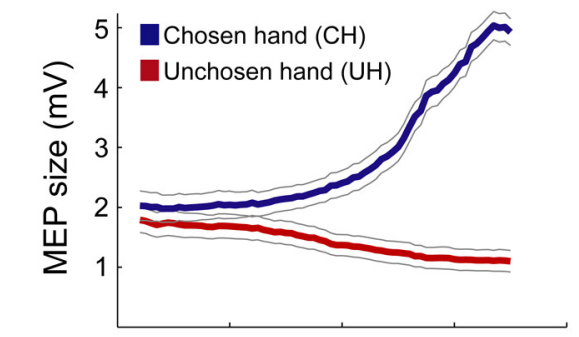

$\mathrm{C} C V_{\text {observed }}$ and $C V_{\text {predicted }}$
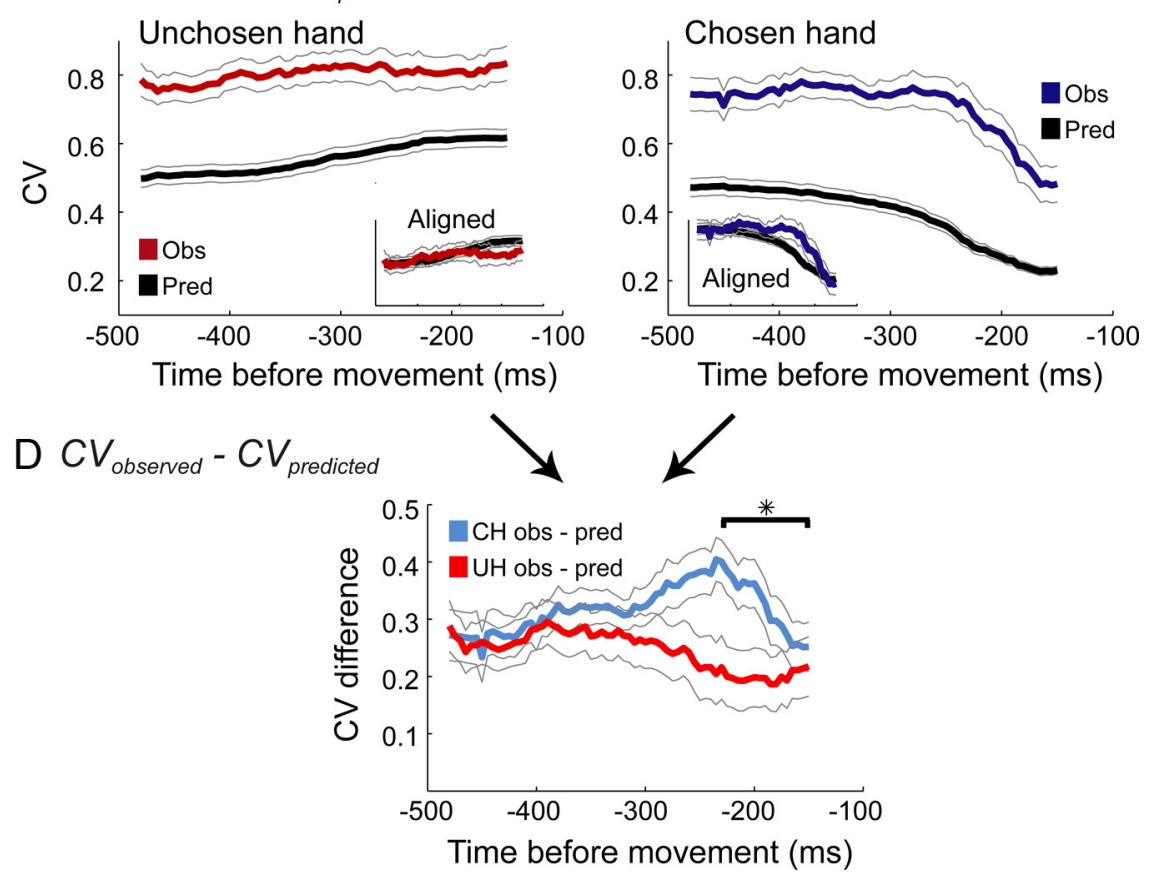

Figure 3. Variability changes in CSE reflect action preparation of the chosen action. $\boldsymbol{A}, \boldsymbol{B}$, Observed mean MEP amplitude $(\boldsymbol{A})$ and SD (B) for right-hand and left-hand responses (i.e., when left M1 facilitates the chosen or inhibits the unchosen action), averaged across choice and forced-choice trials, and plotted over time with respect to movement onset. Because RTs were shorter in forced-choice trials, only the period from $-555 \mathrm{~ms}$ before response is shown (first bin centered at $-480 \mathrm{~ms})$. C, Observed (red, unchosen; blue, chosen) MEP variability (CV) during the task was elevated compared with that predicted based on the model obtained from the resting data (black). The insets show the same data but observed and predicted curves were aligned based on the first $100 \mathrm{~ms}$ of the epoch. $\boldsymbol{D}$, The difference between observed and predicted variability reveals a significant task-related decrease in MEP variability in the last 230 ms before movement onset $\left({ }^{*} p<0.05\right.$ ), which is specific to trials in which the probed hemisphere (here left) facilitated the chosen (here right hand) response. This is consistent with the decrease in variability observed during action preparation in monkey premotor cortex and may thus track how neurons acquire the optimal state for action execution. Error bars indicate SEM.

across participants, TMS times were normalized with respect to the group mean RT, for both choice and forced-choice trials, by multiplying each trial's TMS time by the constant factor: group mean RT/participant mean RT. Consequently, TMS times for all participants with respect to the response were between $[-$ group RT] and $[-\sim 75 \mathrm{~ms}]$.

To obtain continuous measures of MEP size and $\mathrm{CV}_{\text {observed }}$ across the trial, a sliding-window approach (width, $150 \mathrm{~ms}$; step size, $5 \mathrm{~ms}$ ) was applied to the data separately for choice and forced-choice trials and right-hand versus left-hand responses (i.e., when TMS was applied over the M1 facilitating a response with the chosen hand, or inhibiting a response with the unchosen hand, respectively; referred to as "chosen" and "unchosen" responses henceforth). The sliding window covered the time between the mean group RT and $75 \mathrm{~ms}$ before movement onset (i.e., $[-835,-75] \mathrm{ms}$ with respect to movement onset on choice trials, and $[-555,-75] \mathrm{ms}$ in forced-choice trials). This led to 123 bins for choice trials and 67 bins for forced-choice trials, centered between $[-760,-150] \mathrm{ms}$ and $[-480,-150] \mathrm{ms}$, respectively. For every bin, the mean and $\mathrm{CV}_{\text {observed }}$ of all MEPs contained in the corresponding window were calculated. Time windows containing $<5$ MEPs were excluded, thus ensuring bin sizes comparable to the analysis of the resting data and previous studies (e.g., Darling et al., 2006). This only affected time bins immediately before the response, when MEPs were more likely to be rejected for overt muscle activation. The average number of MEPs in each bin was 29.5 (33.6 for choice conditions and 21.9 for forced-choice conditions).

Main analysis 1: overall changes in CSE variability during task performance We first examined the overall effect of task performance on CSE variability for the chosen and unchosen hand. This analysis was conducted on the data merged across choice and forcedchoice trials, so only the final 67 bins contained in both trial types were included. Using the logarithmic model derived from the resting data [see Results (i.e., $\mathrm{CV}_{\text {predicted }}=-0.23 * \log$ $(\mathrm{AMP})+0.59)]$, the $\mathrm{CV}_{\text {observed }}$ of MEPs was compared with the $\mathrm{CV}_{\text {predicted }}$ based on the changes in MEP amplitude. $t$ tests were conducted at every time bin $(n=67)$, and corrected for multiple comparisons using the false discovery rate (FDR) at $p<0.05$ (Benjamini and Hochberg, 1995; Genovese et al., 2002).

We note that it was valid to use the logarithmic function for predicting the temporal evolution of the $\mathrm{CV}_{\text {observed }}$ because it perfectly predicted the time course of observed CSE variability during the first half of a trial (apart from a constant offset) when motor preparatory processes would have been negligible (Fig. $3 C$ ). We therefore defined task-related changes in variability as those occurring over and above the changes predicted based on the model. Because this critically relies on a good model, we also examined the range of model parameters that yield the same statistical results.

\section{Main analysis 2: task-related changes in variability before movement onset}

The variability for chosen versus unchosen responses (merged across choice and forcedchoice conditions) was examined. To isolate task-related changes in variability, we subtracted the predicted from the observed change in CV. Separate $t$ tests were conducted for trials where the right hand was the chosen versus unchosen response hand. The data in the last time bin centered at $-150 \mathrm{~ms}$ (including MEPs measured between $[-225,-75] \mathrm{ms}$ before response) was compared against the data at -230 $\mathrm{ms}$ (interval $[-305,-165] \mathrm{ms})$ [i.e., the time at which the $\mathrm{CV}\left(\mathrm{CV}_{\text {observed }}\right.$ $\left.-\mathrm{CV}_{\text {predicted }}\right)$ started to decrease]. Note the difference in degrees of freedom between unchosen-hand comparisons $(n-1=14)$ and chosen-hand comparisons $(11-1=10)$, which is due to fewer subjects having sufficient useable data for chosen (right hand) responses in the last time bin.

\section{Main analysis 3: behavioral relevance of changes in CSE variability}

We tested whether trials with faster RTs might be preceded by an earlier (or stronger) decline in CSE variability compared with those with slower RTs. In this analysis, we were interested in the action-planning process, but not the decision process required in choice trials. Therefore, we only included forced-choice trials (i.e., when the visual stimulus instructed actions without any ambiguity). Forced-choice trials also had more consistent RTs than choice trials and any differences in RT could be related 
back to the optimization of movement planning, and were not overshadowed, for instance, by a more difficult choice process. We separated fast and slow forced-choice responses using a median split, separately for chosen and unchosen response trials. This yielded equal numbers of trials in the respective fast and slow conditions. Because this also resulted in different average trial durations, the RT of every trial was scaled to 1 (with 0 now denoting trial onset and 1 the time of button press). A slidingwindow approach was used, as described above, to analyze the temporal evolution of changes in MEP size and variability. For this analysis, the step size and window size were slightly larger because only forced-choice trials (33\% of trials) were included, and these were further split into slow and fast trials. The step size in the range $[0,1]$ was 0.02 (corresponding to $\sim 10 \mathrm{~ms}$ ) and the window size 0.35 (corresponding to $\sim 200 \mathrm{~ms}$ ). Windows with $<3$ MEPs were excluded. For each time point, $t$ tests were performed on the $\mathrm{CV}_{\text {observed }}$ (or difference between observed and predicted (CV) of fast and slow trials, separately for each hand, and on chosen versus unchosen responses, separately for fast and slow trials. FDR correction for multiple comparisons was used unless otherwise stated.

\section{Main analysis 4: CSE variability in choice versus}

forced-choice trials

To ascertain whether the additional cognitive processing required in choice trials is reflected in specific changes in CSE variability in either the chosen or unchosen hand (i.e., when the examined hemisphere facilitates the chosen or inhibits the unchosen action, respectively), $t$ tests were used to compare task-related changes in $\mathrm{CV}\left(\mathrm{CV}_{\text {observed }}-\mathrm{CV}_{\text {predicted }}\right)$ between choice and forced choice trials, separately for chosen and unchosen responses.

\section{Root mean square analysis}

Finally, to rule out the possibility that any of our effects might be caused by trials with elevated background EMG, we calculated the root mean square (RMS) of the EMG signal in the $100 \mathrm{~ms}$ before the TMS pulse (Mars et al., 2009). This measure, instead of the MEP amplitude, was then subjected to all analyses and statistical tests reported for MEPs and CVs.

\section{Results}

\section{Relationship between MEP size and variability at rest}

The raw data from all participants are shown in Figure $1 A$. The $\mathrm{CV}$ of the MEPs is plotted as a function of MEP amplitude. Bayesian model comparison revealed that a logarithmic function provided a better fit to the data than a linear function $(\mathrm{BF}=54.5)$. The Bayes factor is the ratio of the log-likelihoods of the linear and logarithmic models. A difference of 54.5 corresponds to $\exp (54.5)=4.6 \mathrm{e} 23$-to- 1 odds, and therefore decisive evidence in favor of the logarithmic model (Bestmann et al., 2008). This result holds for different MEP inclusion criteria, and thus small variations in model fits (all $\mathrm{BF},>4$ ). In Figure $1 A$, the resulting logarithmic fit $\left[\mathrm{CV}_{\text {predicted }}=-0.23^{\star} \log (\mathrm{AMP})+0.59\right]$ is overlaid on the merged data of all participants. The residuals of both the linear and the logarithmic fits are shown in Figure $1 B$.

\section{Changes in CSE variability during action preparation in choice and forced-choice contexts \\ Behavioral results}

Reaction times were significantly longer for choice versus forcedchoice trials (choice, $835 \pm 33 \mathrm{~ms}$; forced choice, $553 \pm 17 \mathrm{~ms}$; $\left.t_{(14)}=10.29, p=6.54 \mathrm{e}-08\right)$, reflecting the time required to make a value decision on choice trials. More details about the behavioral performance on the choice task have been reported previously (Klein-Flügge and Bestmann, 2012).

Variability in CSE is increased during task compared with rest Over the course of a trial, CSE of responses evoked from TMS over left M1 increased for the right-hand (i.e., contralateral) responses and decreased for left-hand (i.e., ipsilateral) responses (Fig. 3A). This difference in MEP amplitude between chosen and unchosen action representations increased progressively toward the overt response, and average changes in CSE thus reflected the prepared versus unprepared action. The change in MEP amplitude was accompanied by changes in MEP SD, as shown in Figure $3 B$.

The predicted change in MEP variability $\left(\mathrm{CV}_{\text {predicted }}\right)$ was obtained for each participant based on the observed changes in MEP amplitude using the logarithmic function described above. Comparing predicted and observed CV showed that variability was consistently higher during task performance compared with rest at all times in a trial $(p<0.05$, FDR-corrected for every time point for both chosen and unchosen responses; Fig. $3 C$ ). Thus, we observed a constant difference between variability at rest and during task performance.

\section{Decreases in CSE variability for the chosen hand immediately before response}

Examining the change in CSE variability across time for the chosen hand revealed a task-related decrease in $\mathrm{CV}\left(\mathrm{CV}_{\text {observed }}-\right.$ $\mathrm{CV}_{\text {predicted }}$ ) immediately before the overt response, meaning this decrease occurred over and above changes in variability expected based on changes in MEP amplitude (bins centered at $-230 \mathrm{~ms}$ vs $-150 \mathrm{~ms} ; \mathrm{CV}_{\text {observed }}-\mathrm{CV}_{\text {predicted }}$ chosen/right hand; $t_{(10)}=$ $4.96, p=5.66 \mathrm{e}-04$; Fig. 3D). From $\sim 230 \mathrm{~ms}$ before the response, the observed pattern for the chosen action representation thus resembled the decrease in variability found in direct recordings from monkey premotor cortex, where firing rates become less variable as the monkey prepares to move (Churchland et al., 2006a).

This result relies on a good model for $\mathrm{CV}_{\text {predicted }}$ because $\mathrm{CV}_{\text {predicted }}$ is subtracted from $\mathrm{CV}_{\text {observed }}$ Importantly, the result was robust to any change in the intercept parameter of $\mathrm{CV}_{\text {predicted }}$, and held true for slope parameters in the range $[-0.5$,infinity]. Thus, our conclusions were not dependent on the exact format of the model used to determine $\mathrm{CV}_{\text {predicted }}$.

There are three additional observations. First, the task-related decrease in $\mathrm{CV}\left(\mathrm{CV}_{\text {observed }}-\mathrm{CV}_{\text {predicted }}\right)$ was not observed when the left hand was used to make the response, i.e., when the stimulated hemisphere did not facilitate the chosen action (bins centered at $-230 \mathrm{~ms}$ vs $-150 \mathrm{~ms} ; \mathrm{CV}_{\text {observed }}-\mathrm{CV}_{\text {predicted }}$ unchosen/ left hand; $\left.t_{(14)}=-0.09, p=0.93\right)$. Second, for the chosen response, variability started decreasing $\sim 80 \mathrm{~ms}$ later than predicted based on the logarithmic model (Fig. 3C), so that the difference between observed and predicted $\mathrm{CV}$ showed an increase in variability that preceded the later decrease (Fig. 3D). Third, despite the unspecific increase in variability during task performance, the dynamics of CSE variability for the initial half of a trial were correctly predicted by the relationship characterized at rest, as shown by a stable difference between predicted and observed CV up until $\sim 300 \mathrm{~ms}$ before movement onset (Fig. 3D).

Behavioral relevance of decrease in CSE variability of chosen hand To examine whether the decline in CSE variability observed immediately before movement onset was behaviorally meaningful for the execution of the action, we separately examined the faster and slower half of trials. Based on findings in nonhuman primates (Churchland et al., 2006a), we expected that faster responses may be facilitated by lower premovement variability, which reflects a more consistent neuronal state. Because we were interested in action planning and preparation, as opposed to the choice process itself, this analysis was restricted to forced-choice trials. In forced-choice trials, there was no ambiguity about the response because the action was explicitly instructed, and thus any differences in $\mathrm{RT}$ across trials can be more readily attributed 
A
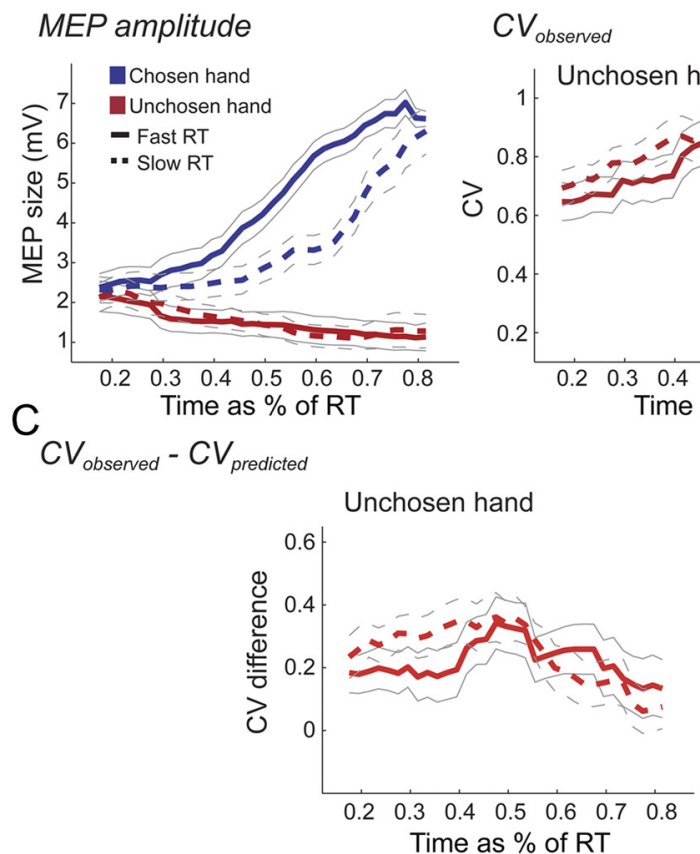

B
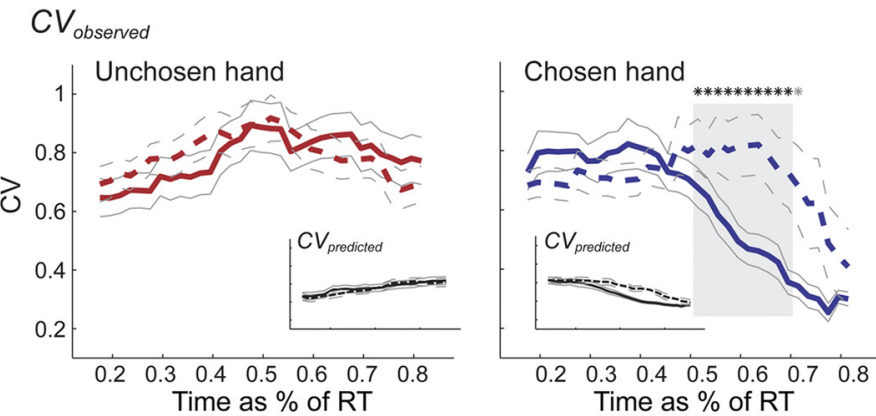

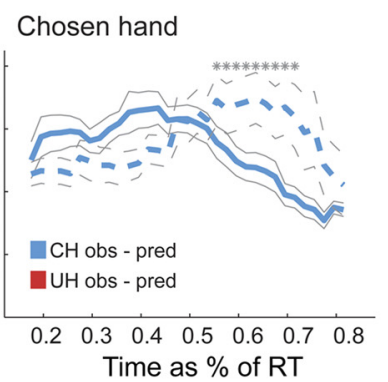

Figure 4. Decreases in CSE variability distinguish between slow and fast actions. $\boldsymbol{A}$, Observed mean MEP amplitude for the chosen and unchosen hand, separately for fast (continuous) and slow (dotted) forced-choice trials. Because of the different average trial duration of slow and fast trials, the RT of every trial was scaled to 1 (with 0 now denoting trial onset and 1 the time of button press). $\boldsymbol{B}$, Variability (CV) decreases earlier on fast compared with slow trials for the chosen hand, but not for the unchosen hand (black stars, FDR-corrected $p<0.05$; gray stars, uncorrected $p<0.05$; gray area highlights area with FDR-corrected significance). Insets show the expected CV (i.e., the $\mathrm{CV}_{\text {predicted }}$ based on observed changes in MEP size) for the chosen versus unchosen hand for direct comparison with the changes actually observed. $C$, The difference between $\mathrm{CV}_{\text {observed }}$ and $\mathrm{CV}_{\text {predicted }}$ shown separately for trials with fast and slow responses. Decreases in $C V$ for responses with the chosen hand occur over and above those predicted based on intrinsic CSE variability, both on slow and fast trials, and are thus task-related. Importantly, the task-related portion of the $\mathrm{CV}_{\text {( }} \mathrm{CV}_{\text {observed }}$ $-\mathrm{CV}_{\text {predicted }}$ ) decreases earlier in fast-response trials (gray stars, uncorrected $p<0.05$ ), compared with slow-response trials. Changes in variability are thus directly related to behavior and the optimization of the prepared action representation. Error bars indicate SEM.

to differences in the optimization of action planning. The average RT on fast compared with slow forced-choice trials was $453 \pm 11$ $\mathrm{ms}$ and $625 \pm 23 \mathrm{~ms}$, respectively. To allow for comparison of trials with such different RTs, the RT of every trial was scaled to 1 (with 0 denoting trial onset, and 1 the time of button press).

CSE increased faster for fast compared with slow responses in the chosen hand (Fig. 4A). Furthermore CSE variability was indeed found to be smaller preceding a fast compared with a slow response $(p<0.05$, FDR-corrected on 10 consecutive data points between 52 and $70 \%$ of the trial's RT; Fig. $4 B$, right). For the unchosen hand, no such difference between the $\mathrm{CV}_{\text {observed }}$ in fast-response versus slow-response trials was observed $(p>0.05$ for all time points; Fig. $4 B$, left). While for fast responses, the $\mathrm{CV}_{\text {observed }}$ of the chosen hand already decreased with respect to the $\mathrm{CV}_{\text {observed }}$ of the unchosen hand in this time window (8 of these 10 data points, $p<0.05$, FDR-corrected), this was not the case for slow responses (all $p>0.05$ ). For slow responses, only the last three time points of the $\mathrm{CV}_{\text {observed }}$ of the chosen and unchosen hand (corresponding to $77-82 \%$ of the trial's RT) were significantly different when using FDR correction $(p<0.05)$. This was true for the last 13 time points (i.e., 57-82\%) for fast responses. To verify that this earlier variability decrease in fastresponse trials was task-related, we calculated the difference between observed and predicted CV (Fig. 4C). This revealed a more pronounced and earlier task-related decrease in fast compared with slow trials in the chosen hand; however, this effect did not reach FDR-corrected significance (smallest uncorrected $p$ value, $p=0.0061$ at $64 \%$; Fig. $4 C$ ). We note, however, that this analysis had reduced power because forced-choice trials make up only $33 \%$ of trials, which are further reduced during preprocess- ing, and split into four conditions here. Thus, each time course (Fig. $4 A-C$ ) is derived from only $\sim 7 \%$ of trials. Nevertheless, nine consecutive values between 55 and $72 \%$ of the trial's $\mathrm{RT}$ reached uncorrected significance in the chosen hand for $\mathrm{CV}_{\text {observed }}-\mathrm{CV}_{\text {predicted }}($ all $p<0.035$, uncorrected).

Choice-specific changes in CSE variability in the unchosen hand In a final analysis, we asked whether the different cognitive processes involved in choice and forced-choice trials differentially affected the underlying CSE variability. Comparing the CV $\left(\mathrm{CV}_{\text {observed }}-\mathrm{CV}_{\text {predicted }}\right)$ in the chosen hand for choice versus forced-choice trials revealed no significance differences (all $p>$ 0.05; Fig. 5, right). However, in the unchosen hand, there was a significant difference between choice and forced-choice trials $(p<0.05$, uncorrected at 19 consecutive time bins with bin centers in the range $[-320,-230] \mathrm{ms}$; Fig. 5, left). Further examination of the observed effect revealed that it was driven by an earlier-than-expected decrease in variability in choice trials in the unchosen hand (Fig. 5). This suggests that the additional processing required during a value-based decision results in more consistent neuronal states. This effect is absent when a response is directly instructed. The increased consistency seems to be specific to the population representing the unchosen action and is thus likely to reflect inhibitory processes mediated by other regions that converge on $\mathrm{M} 1$.

Effects are not tied to exact form of logarithmic relationship Changes in CSE variability predicted based on the logarithmic relationship determined at rest $\left[\mathrm{CV}_{\text {predicted }}=-0.23^{\star} \log (\mathrm{AMP})+0.59\right]$ were subtracted from the observed CSE variability $\left(\mathrm{CV}_{\text {observed }}\right)$ in 
all main analyses (Figs. 3D, 4C, 5). To test the robustness of our findings against variations of this function, we repeated these analyses with slightly modified fits, which we obtained by including a different range of MEP amplitudes (see Materials and Methods). By definition, changes in the intercept parameter had no effect on any results because an upward or downward shift of all CV time courses does not affect their differential relationship. The changes in the slope parameter from these alternative fits (range, $-0.18-$ -0.24 ) all resulted in the same statistical results, showing that our conclusions do not rely on the exact form of the logarithmic function. Note that even larger deviations up to -0.4 for the slope still allow us to draw the same conclusions.

\section{$\mathrm{CV}_{\text {observed }}-\mathrm{CV}_{\text {predicted }}$ for choice versus forced choice trials}
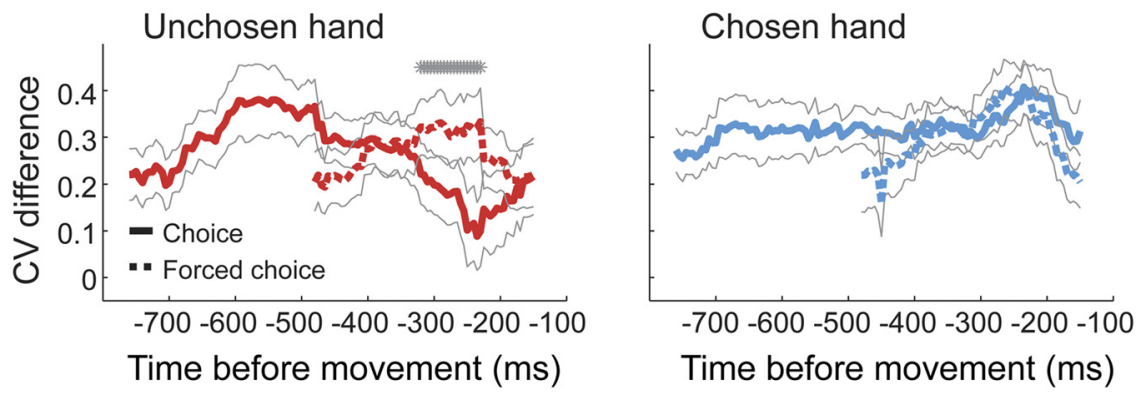

Time before movement (ms)

Figure 5. Earlier decrease of variability for unchosen hand in choice trials but not in forced-choice trials. The difference between $\mathrm{CV}_{\text {observed }}$ and $\mathrm{CV}_{\text {predicted }}$ is displayed separately for choice and forced-choice trials. For the chosen hand, MEP variability does not differ between choice and forced-choice trials (right). For the unchosen hand, however, there is a significant difference between choice and forced-choice trials between 320 and 230 ms preceding the response (gray stars, $p<0.05$, uncorrected). This difference is driven by an early decrease in variability for unchosen actions (here, left-hand responses) present only in choice trials. Together, this suggests that the inhibition of an unchosen action resulting from a choice process also leads to diminished across-trial variability and thus a more consistent neuronal state. Error bars indicate SEM.

Elevated background EMG does not explain the observed changes in CSE variability

Trials with muscle precontraction in the FDI were discarded from all analyses. To further ensure that none of our effects were caused by subthreshold fluctuations in background EMG, we repeated all analyses and statistical tests on the RMS (see Materials and Methods). None of the statistical tests reported in the manuscript were significant when performed on the mean RMS instead of the mean/CV of the MEPs, showing that muscle precontraction was not driving any of our effects.

\section{Discussion}

We report changes in the variability of human CSE before a movement that are specific to the chosen action. This shows that CSE variability can provide insight into the preparatory state of the motor system, analogous to changes in firing-rate variability during action preparation in monkeys (Churchland et al., 2006a).

\section{State effects of variability: task versus rest}

We first characterized the relationship between CSE and variability at rest. This provided a detailed model of intrinsic CSE variability and allowed us to identify any additional process-related changes in CSE variability during task performance. When comparing CSE variability during task performance with that found for equivalent MEP amplitudes at rest, we found an overall variability increase present throughout the trial. Heightened levels of firing-rate variability have similarly been reported in a matchingpennies choice task in nonhuman primates (Lee and Seo, 2011), even though this study focused on very different regions (prefrontal/parietal) and processes (stimulus-evoked and outcomeevoked changes in variability rather than action preparation). There are several potential explanations for this effect: increased levels of alertness during task performance may lead to elevated CSE and may vary across trials, and there was variability in the presented behavioral events, such as varying probabilities and magnitudes, which can explain a large proportion of trial-by-trial variability in neural firing rates (Lee and Seo, 2011). Importantly, the heightened variability is a general effect of state and independent of specific motor preparatory processes of main interest in this study.

\section{Variability decline during action preparation supports optimal subspace hypothesis}

Our main finding was a decrease in CSE variability from $\sim 230 \mathrm{~ms}$ before movement, which was specific to the responding hand. Importantly, this effect was task-related in that it occurred over and above the variability changes trivially expected based on the concurrent rise in CSE accompanying motor preparation. This confirmed our main hypothesis showing that read-outs of the variability of CSE obtained at a population level in humans using TMS over M1 (i.e., the MEP) can track the consistency of the state of preparation. Work in nonhuman primates has previously established that changes in variability can provide meaningful insights into the state of motor cortex (Churchland et al., 2006a; Mandelblat-Cerf et al., 2009; Rickert et al., 2009). It has been suggested that the increasingly consistent firing rates in monkey premotor cortex during action preparation (Churchland et al., 2006a) reflect the system's approach to an optimal state for a particular movement (Churchland et al., 2006b; Churchland and Shenoy, 2007; Rickert et al., 2009). The optimization process is thought to produce a movement with the desired properties when firing rates converge to a specific state, with different optimal states for different movements (Churchland et al., 2010b; Afshar et al., 2011; Shenoy et al., 2011; "optimal subspace hypothesis”). This optimization process should thus be specific to the performed action. In the present study, it should therefore be observed only in right-hand response trials where the stimulated left hemisphere enabled execution of the action. Indeed, CSE became increasingly consistent for the chosen (right) but not for the unchosen (left) hand, at a time scale consistent with direct recordings (Churchland et al., 2006a).

Interestingly, the decrease in CSE variability for the chosen response started later than was predicted from the concurrent rise in MEP amplitude. The difference between observed and predicted CV thus revealed a task-related variability increase that preceded the subsequent decline. This observation is striking in that the variability increase occurs around the time when many critical task-related inputs are likely to converge onto M1. In our task, such inputs differ on a trial-by-trial basis because the offered values change, which in turn will affect cortical (and possibly spinal) structures to varying degrees across different trials. Previous data support the idea that the information encoded by neurons is conveyed through their trial-by-trial variability (Scaglione 
et al., 2011). While the motivation to perform the chosen action will vary across trials in our task, the action itself will be consistent across trials; this could explain the co-occurrence of both an increase and a decrease in task-related variability we observe.

\section{Possible sources of changes in variability of MEPs}

Previous nonhuman primate work showed decreases in firingrate variability during action preparation in dorsal premotor cortex (PMd; Churchland et al., 2006a). Here we report similar effects in humans, albeit at a population level. Of importance here is that the MEP measured in our study is a (smoothed) compound signal of several descending corticospinal volleys, including an initial subcortical D-wave and several subsequent I-waves (Di Lazzaro et al., 2008). While the initial D-wave and first I-wave are practically resistant to changes by any cognitive manipulation, later I-waves are largely under the influence from transcortical (likely to be premotor or postcentral) inputs into M1 at latencies between 2.4 and $7 \mathrm{~ms}$ (Di Lazzaro et al., 2008), and are readily amenable to cognitive influences (Bestmann, 2012). This means that the present MEP effects are unlikely to be driven purely by changes at the spinal level, though this cannot be ruled out based on the current data and should be addressed in future work. Instead, they more likely originate from premotor inputs into M1, with PMd being a likely candidate region given its anatomical location (Dum and Strick, 1991; Tomassini et al., 2007) and functional role in action preparation (e.g., Schluter et al., 1998; Pastor-Bernier et al., 2012).

\section{Variability changes after trial onset}

Task-related decreases in variability are not specific to the motor system. Similar variability changes have been observed in several cortical regions and in a variety of tasks following presentation of a sensory stimulus, suggesting that sensory input stabilizes neuronal firing rates (Churchland et al., 2010a and references therein). Here, we did not observe such a stimulus-locked decrease in CSE variability, which could have several explanations. First, our design was not optimized for that time window because sampling of CSE was sparse close to trial onset. Second, our visual stimuli differed on a trial-by-trial basis in terms of their associated probabilities and magnitudes, resulting in different levels of motivation and choice difficulty. This introduced additional variability that may have masked stimulus-evoked decreases in variability. It also meant that action preparation was not very consistent early in the trial. Similarly, Churchland et al. (2006a, Fig. 6C) did not observe stimulus-evoked decreases in variability when less emphasis was placed on consistent action preparation early on. Finally, the CSE measure recorded in our experiment might be less sensitive to stimulus-evoked influences. When comparing different brain regions, the relative variability decline after stimulus onset indeed seems to be most pronounced in visual brain regions (V1, V4, MT), but less so in orbitofrontal or premotor regions (Churchland et al., 2010a, Fig. 3). Consistent with this, it has been suggested that the time course of variability relates to the information encoded by the neurons in that particular brain region (Lee and Seo, 2011; Scaglione et al., 2011).

\section{Behavioral relevance of temporal changes in variability}

The increased consistency of the neuronal state before a response is thought to bear behavioral relevance for optimizing action preparation. For example, in premotor cortex and the frontal eye fields, firing-rate variability correlates with reaction time (Churchland et al., 2006a; Cohen et al., 2007; Steinmetz and Moore, 2010; Purcell et al., 2012), and variability declines as more information about the upcoming movement is revealed (Rickert et al., 2009). Similarly, variability in other brain regions relates to task aspects, such as attention (Cohen and Maunsell, 2009) and learning (Mandelblat-Cerf et al., 2009). Here we found an earlier variability decrease in trials with fast compared with slow responses in the chosen hand, confirming that the observed changes in variability before movement onset are also behaviorally meaningful at the population level.

\section{Variability changes reflect choice-specific processes}

Finally, our data also revealed an earlier decrease in variability for the unchosen hand that was specific to choice trials. Choices in a visual motion task can be predicted based on changes in the variability of neuronal responses in monkey area MT (Britten et al., 1996). However, this is the first time variability could be compared between choice and forced-choice contexts. Choice processes are thought to facilitate action selection in motor regions through biased competition (Cisek, 2006; Pastor-Bernier and Cisek, 2011), which is fuelled by inputs from other regions. Indeed, such signatures of choice-based competition can modulate CSE (Klein-Flügge and Bestmann, 2012), but whether choice processes might similarly be tracked by changes in variability has thus far not been investigated. Consistent with the idea that a decline in variability reflects an optimization process, the decline in CSE variability for unselected actions observed here may reflect how an increased consistency in the neuronal state is achieved through biasing inhibitory processes. This optimization process would be expected to start earlier with respect to the response in choice compared with forced-choice contexts, which is consistent with our finding.

\section{Validity of TMS as a novel approach for studying changes in CSE variability in humans}

While our results are congruent with recent reports from nonhuman primates, there are notable differences in the measures used here compared with those obtained with direct recordings. Precisely how these two measures relate remains unclear, but we note that this applies to all human TMS-M1 studies, not just the variability-based analyses reported here. Single-unit recordings measure variability in spiking across trials in the same neuron (often for many neurons). Instead, we here used noninvasive techniques to find out whether variability of a population response can predict the state of action preparation, and thus whether the changes observed at the single-unit level can also be seen at the population level in humans. Both theoretical work (Sussillo and Abbott, 2009; Abbott et al., 2011) and data from direct recordings (Churchland et al., 2010a) confirm that the previously observed decline in neural variability reflects a network property. Indeed, to be functionally relevant, a large proportion of neurons must exhibit similar reductions in firing-rate variability during action preparation, and exciting this population at once using TMS will similarly yield less-variable output. To the best of our knowledge, our study is the first demonstration in humans that the evolution of CSE variability tracks action preparation and may also reflect influences from more complex processes, such as decision making.

\section{References}

Abbott L, Rajan K, Sompolinksy H (2011) Interactions between intrinsic and stimulus-dependent activity in recurrent neural networks. In: The dynamic brain: an exploration of neuronal variability and its functional significance (Ding M, Glanzman D, eds), pp 65-82. New York: Oxford UP.

Afshar A, Santhanam G, Yu BM, Ryu SI, Sahani M, Shenoy KV (2011) 
Single-trial neural correlates of arm movement preparation. Neuron 71: 555-564. CrossRef Medline

Benjamini Y, Hochberg Y (1995) Controlling the false discovery rate: a practical and powerful approach to multiple testing. J Royal Stat Soc Series B (Methodological) 57:289-300.

Bestmann S (2012) Functional modulation of primary motor cortex during action selection. In: Cortical connectivity (Chen R, Rothwe JC, eds), pp 183-206. Berlin: Springer.

Bestmann S, Harrison LM, Blankenburg F, Mars RB, Haggard P, Friston KJ, Rothwell JC (2008) Influence of uncertainty and surprise on human corticospinal excitability during preparation for action. Curr Biol 18:775780. CrossRef Medline

Britten KH, Newsome WT, Shadlen MN, Celebrini S, Movshon JA (1996) A relationship between behavioral choice and the visual responses of neurons in macaque MT. Vis Neurosci 13:87-100. CrossRef Medline

Capaday C, Lavoie BA, Barbeau H, Schneider C, Bonnard M (1999) Studies on the corticospinal control of human walking. I. Responses to focal transcranial magnetic stimulation of the motor cortex. J Neurophysiol 81:129-139. Medline

Churchland MM, Shenoy KV (2007) Delay of movement caused by disruption of cortical preparatory activity. J Neurophysiol 97:348-359. CrossRef Medline

Churchland MM, Yu BM, Ryu SI, Santhanam G, Shenoy KV (2006a) Neural variability in premotor cortex provides a signature of motor preparation. J Neurosci 26:3697-3712. CrossRef Medline

Churchland MM, Afshar A, Shenoy KV (2006b) A central source of movement variability. Neuron 52:1085-1096. CrossRef Medline

Churchland MM, Yu BM, Cunningham JP, Sugrue LP, Cohen MR, Corrado GS, Newsome WT, Clark AM, Hosseini P, Scott BB, Bradley DC, Smith MA, Kohn A, Movshon JA, Armstrong KM, Moore T, Chang SW, Snyder LH, Lisberger SG, Priebe NJ, et al. (2010a) Stimulus onset quenches neural variability: a widespread cortical phenomenon. Nat Neurosci 13:369-378. CrossRef Medline

Churchland MM, Cunningham JP, Kaufman MT, Ryu SI, Shenoy KV (2010b) Cortical preparatory activity: representation of movement or first cog in a dynamical machine? Neuron 68:387-400. CrossRef Medline

Cisek P (2006) Integrated neural processes for defining potential actions and deciding between them: a computational model. J Neurosci 26: 9761-9770. CrossRef Medline

Cohen JY, Pouget P, Woodman GF, Subraveti CR, Schall JD, Rossi AF (2007) Difficulty of visual search modulates neuronal interactions and response variability in the frontal eye field. J Neurophysiol 98:2580-2587. CrossRef Medline

Cohen MR, Maunsell JH (2009) Attention improves performance primarily by reducing interneuronal correlations. Nat Neurosci 12:1594-1600. CrossRef Medline

Darling WG, Wolf SL, Butler AJ (2006) Variability of motor potentials evoked by transcranial magnetic stimulation depends on muscle activation. Exp Brain Res 174:376-385. CrossRef Medline

Devanne H, Lavoie BA, Capaday C (1997) Input-output properties and gain changes in the human corticospinal pathway. Exp Brain Res 114:329-338. CrossRef Medline

Di Lazzaro V, Ziemann U, Lemon RN (2008) State of the art: physiology of transcranial motor cortex stimulation. Brain Stimul 1:345-362. CrossRef Medline

Dum RP, Strick PL (1991) The origin of corticospinal projections from the premotor areas in the frontal lobe. J Neurosci 11:667-689. Medline

Duque J, Ivry RB (2009) Role of corticospinal suppression during motor preparation. Cereb Cortex 19:2013-2024. CrossRef Medline

Duque J, Lew D, Mazzocchio R, Olivier E, Ivry RB (2010) Evidence for two concurrent inhibitory mechanisms during response preparation. J Neurosci 30:3793-3802. CrossRef Medline

Evarts EV (1966) Pyramidal tract activity associated with a conditioned hand movement in the monkey. J Neurophysiol 29:1011-1027. Medline

Faisal AA, Selen LP, Wolpert DM (2008) Noise in the nervous system. Nat Rev Neurosci 9:292-303. CrossRef Medline

Genovese CR, Lazar NA, Nichols T (2002) Thresholding of statistical maps in functional neuroimaging using the false discovery rate. Neuroimage 15:870-878. CrossRef Medline

Kass RE, Raftery AE (1995) Bayes factors. J Am Stat Assoc 90:773-795. CrossRef

Kiers L, Cros D, Chiappa KH, Fang J (1993) Variability of motor potentials evoked by transcranial magnetic stimulation. Electroencephalogr Clin Neurophysiol 89:415-423. CrossRef Medline

Klein-Flügge MC, Bestmann S (2012) Time-dependent changes in human corticospinal excitability reveal value-based competition for action during decision processing. J Neurosci 32:8373-8382. CrossRef Medline

Lee D, Seo H (2011) Neural and behavioral variability related to stochastic choices during a mixed-strategy game. In: Dynamic brain (Ding M, Glanzman DL, ed), pp 255-275. Oxford: Oxford UP.

Leocani L, Cohen LG, Wassermann EM, Ikoma K, Hallett M (2000) Human corticospinal excitability evaluated with transcranial magnetic stimulation during different reaction time paradigms. Brain 123:1161-1173. CrossRef Medline

Mandelblat-Cerf Y, Paz R, Vaadia E (2009) Trial-to-trial variability of single cells in motor cortices is dynamically modified during visuomotor adaptation. J Neurosci 29:15053-15062. CrossRef Medline

Mars RB, Bestmann S, Rothwell JC, Haggard P (2007) Effects of motor preparation and spatial attention on corticospinal excitability in a delayed-response paradigm. Exp Brain Res 182:125-129. CrossRef Medline

Mars RB, Klein MC, Neubert FX, Olivier E, Buch ER, Boorman ED, Rushworth MF (2009) Short-latency influence of medial frontal cortex on primary motor cortex during action selection under conflict. J Neurosci 29:6926-6931. CrossRef Medline

Miller P, Katz DB (2010) Stochastic transitions between neural states in taste processing and decision-making. J Neurosci 30:2559-2570. CrossRef Medline

Mitchell JF, Sundberg KA, Reynolds JH (2007) Differential attentiondependent response modulation across cell classes in macaque visual area V4. Neuron 55:131-141. CrossRef Medline

Pastor-Bernier A, Cisek P (2011) Neural correlates of biased competition in premotor cortex. J Neurosci 31:7083-7088. CrossRef Medline

Pastor-Bernier A, Tremblay E, Cisek P (2012) Dorsal premotor cortex is involved in switching motor plans. Front Neuroeng 5:5. CrossRef Medline

Purcell BA, Heitz RP, Cohen JY, Schall JD (2012) Response variability of frontal eye field neurons modulates with sensory input and saccade preparation but not visual search salience. J Neurophysiol 108:2737-2750. CrossRef Medline

Rickert J, Riehle A, Aertsen A, Rotter S, Nawrot MP (2009) Dynamic encoding of movement direction in motor cortical neurons. J Neurosci 29: 13870-13882. CrossRef Medline

Rosner R, Warzecha AK (2011) Relating neuronal to behavioral performance: variability of optomotor responses in the blowfly. PLoS ONE 6:e26886. CrossRef Medline

Rossi S, Hallett M, Rossini PM, Pascual-Leone A, Pascual-Leone A (2009) Safety, ethical considerations, and application guidelines for the use of transcranial magnetic stimulation in clinical practice and research. Clin Neurophysiol 120:2008-2039. CrossRef Medline

Scaglione A, Moxon KA, Aguilar J, Foffani G (2011) Trial-to-trial variability in the responses of neurons carries information about stimulus location in the rat whisker thalamus. Proc Natl Acad Sci U S A 108:14956-14961. CrossRef Medline

Schluter ND, Rushworth MF, Passingham RE, Mills KR (1998) Temporary interference in human lateral premotor cortex suggests dominance for the selection of movements. A study using transcranial magnetic stimulation. Brain 121:785-799. CrossRef Medline

Shadlen MN, Newsome WT (1998) The variable discharge of cortical neurons: implications for connectivity, computation, and information coding. J Neurosci 18:3870-3896. Medline

Shenoy KV, Kaufman MT, Sahani M, Churchland MM (2011) A dynamical systems view of motor preparation: implications for neural prosthetic system design. Prog Brain Res 192:33-58. CrossRef Medline

Smith AE, Sale MV, Higgins RD, Wittert GA, Pitcher JB (2011) Male human motor cortex stimulus-response characteristics are not altered by aging. J Appl Physiol 110:206-212. CrossRef Medline

Starr A, Caramia M, Zarola F, Rossini PM (1988) Enhancement of motor cortical excitability in humans by non-invasive electrical stimulation appears prior to voluntary movement. Electroencephalogr Clin Neurophysiol 70:26-32. CrossRef Medline

Steinmetz NA, Moore T (2010) Changes in the response rate and response variability of area V4 neurons during the preparation of saccadic eye movements. J Neurophysiol 103:1171-1178. CrossRef Medline 
Sussillo D, Abbott LF (2009) Generating coherent patterns of activity from chaotic neural networks. Neuron 63:544-557. CrossRef Medline

Tomassini V, Jbabdi S, Klein JC, Behrens TE, Pozzilli C, Matthews PM, Rushworth MF, Johansen-Berg H (2007) Diffusion-weighted imaging tractography-based parcellation of the human lateral premotor cortex identifies dorsal and ventral subregions with anatomical and functional specializations. J Neurosci 27:10259-10269. CrossRef Medline

van Elswijk G, Kleine BU, Overeem S, Stegeman DF (2007) Expectancy induces dynamic modulation of corticospinal excitability. J Cogn Neurosci 19:121-131. CrossRef Medline 